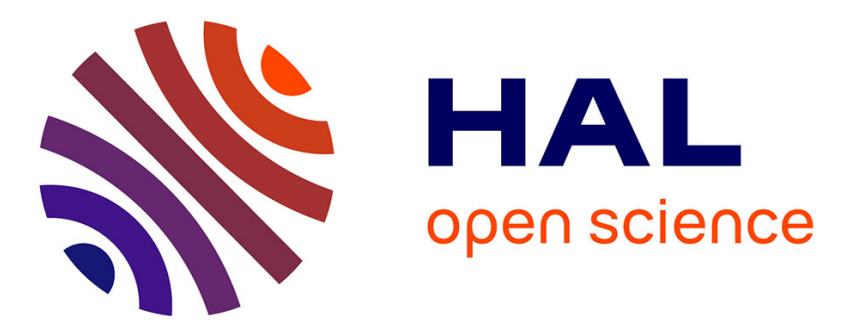

\title{
Singular Perturbation Approximation of Linear Hyperbolic Systems of Balance Laws
}

\author{
Ying Tang, Christophe Prieur, Antoine Girard
}

\section{To cite this version:}

Ying Tang, Christophe Prieur, Antoine Girard. Singular Perturbation Approximation of Linear Hyperbolic Systems of Balance Laws. IEEE Transactions on Automatic Control, 2016, 61 (10), pp.3031-3037. 10.1109/TAC.2015.2499444 . hal-01276260

\section{HAL Id: hal-01276260 https://hal.science/hal-01276260}

Submitted on 19 Feb 2016

HAL is a multi-disciplinary open access archive for the deposit and dissemination of scientific research documents, whether they are published or not. The documents may come from teaching and research institutions in France or abroad, or from public or private research centers.
L'archive ouverte pluridisciplinaire HAL, est destinée au dépôt et à la diffusion de documents scientifiques de niveau recherche, publiés ou non, émanant des établissements d'enseignement et de recherche français ou étrangers, des laboratoires publics ou privés. 


\title{
Singular perturbation approximation of linear hyperbolic systems of balance laws
}

\author{
Ying Tang, Christophe Prieur, and Antoine Girard
}

\begin{abstract}
This paper deals with a class of linear hyperbolic systems of balance laws with multiple time scales. The scale of time constants is modeled by a perturbation parameter. This parameter is introduced in both dynamics and boundary conditions. The solution of the full system is approximated by that of the reduced subsystem when the perturbation parameter is small enough. Lyapunov technique is used to prove it. The main result is illustrated by an academic example. Moreover, the boundary control synthesis to a gas flow transport model is shown based on singular perturbation approach.
\end{abstract}

keywords Linear hyperbolic system, Balance law, Singular perturbation method, Lyapunov technique

\section{INTRODUCTION}

Singular perturbation techniques were introduced in control of finite dimensional systems in late 1960s and became a powerful tool for control design [9], [10], [11], [12]. A class of infinite dimensional singularly perturbed hyperbolic systems has been studied in [19], [16]. Many distributed physical systems can be described by such systems, for instance, gas flow in pipelines [4], [6], hydraulic networks [1], electrical transmission networks [5] or road traffic networks [7].

This paper focuses on a class of linear hyperbolic systems of balance laws where the perturbation parameter $\epsilon$ is introduced in both dynamics and boundary conditions. The first contribution of this paper is the Tikhonov approximation of linear hyperbolic system with source term. More precisely, the solution of the full system can be approximated by that of the reduced subsystem when the perturbation parameter is sufficiently small. This is proved by a Lyapunov function. To the best of our knowledge, this is the first paper dealing with such systems. An academic example is used to illustrate the main result. The second contribution is the boundary control synthesis for application to a gas transport model where the slow dynamics is stabilized in finite time. This system is written as a singularly perturbed model where the transport velocities depend on $\epsilon$ that is different to our previous work [19]. In that work, a class of linear hyperbolic system of conservation laws has been studied and a different approach has been used to model the gas transport system where the transport velocities are constant values.

Y. Tang is with Centre de Recherche en Automatique de Nancy, 2 avenue de la forêt de Haye, 54516 Vandoeuvre-les-Nancy, France. ying.tang@univ-lorraine.fr.

C. Prieur is with the Department of Automatic Control, Gipsa-lab, Université de Grenoble, 11 rue des Mathématiques, BP 46, 38402 Saint-Martin d'Hères Cedex, France. christophe.prieur@gipsa-lab.fr.

A. Girard is with Laboratoire des signaux et systmes (L2S), CNRS, CentraleSuplec, Universit Paris-Sud, Universit Paris-Saclay, 3 rue Joliot-Curie, 91192 Gif-sur-Yvette, Cedex, France. Antoine.Girardel2s.centralesupelec.fr
The paper is organized as follows. Section II presents the full system and the reduced subsystem under consideration. The Tikhonov approximation is given in Section III. Section IV shows the statement of the proof of the Tikhonov theorem. In Section V we first use an academic example to illustrate the general main result. Then, a physical application to a gas flow transport model is shown in the same section. The conclusions are given in Section VI. Due to space limitation, some proofs have been omitted and given in [17].

Notation. Given a matrix $A \in \mathbb{R}^{m \times m}, A^{-1}$ and $A^{\top}$ represent the inverse and the transpose matrix of $A$ respectively. The minimum and maximum eigenvalues of the matrix $A$ are denoted by $\underline{\lambda}(A)$ and $\bar{\lambda}(A)$. For a positive integer $n, I_{n}$ is the identity matrix in $\mathbb{R}^{n \times n} \cdot|\cdot|$ denotes the usual Euclidean norm in $\mathbb{R}^{n}$ and $\|\cdot\|$ is associated with the matrix norm. $\|\cdot\|_{L^{2}}$ denotes the associated norm in $L^{2}(0,1)$ space, defined by $\|f\|_{L^{2}}=\sqrt{\int_{0}^{1}|f(x)|^{2} d x}$ for all functions $f \in L^{2}(0,1)$. Similarly, The associated norm in $H^{2}(0,1)$ space is denoted by $\|\cdot\|_{H^{2}}$, defined for all functions $f \in H^{2}(0,1)$, by $\|f\|_{H^{2}}=\sqrt{\int_{0}^{1}\left(|f(x)|^{2}+\left|f^{\prime}(x)\right|^{2}+\left|f^{\prime \prime}(x)\right|^{2}\right) d x}$. According to [3], for all matrices $G \in \mathbb{R}^{n \times n}, \rho_{1}(G)=\inf \left\{\left\|\Delta G \Delta^{-1}\right\|, \Delta \in\right.$ $\left.D_{n,+}\right\}$, where $D_{n,+}$ denotes the set of diagonal positive matrices in $\mathbb{R}^{n \times n}$.

\section{SYSTEM DESCRIPTION}

Consider the following linear hyperbolic system of balance laws

$$
\begin{aligned}
y_{t}(x, t)+\Lambda_{1}(\epsilon) y_{x}(x, t) & =a(\epsilon) y(x, t)+b(\epsilon) z(x, t), \\
\epsilon z_{t}(x, t)+\Lambda_{2}(\epsilon) z_{x}(x, t) & =c(\epsilon) y(x, t)+d(\epsilon) z(x, t),
\end{aligned}
$$

where $x \in[0,1], t \in[0,+\infty) . \Lambda_{1}(\epsilon)$ is a diagonal matrix in $\mathbb{R}^{n \times n}$ such that $\Lambda_{1}(\epsilon)=\operatorname{diag}\left(\lambda_{1}(\epsilon), \cdots, \lambda_{n}(\epsilon)\right)$, where the $i$ first elements are negative and the $n-i$ last elements are positive. Similarly $\Lambda_{2}(\epsilon)$ is a diagonal matrix in $\mathbb{R}^{m \times m}$, such that $\Lambda_{2}(\epsilon)=\operatorname{diag}\left(\lambda_{1}(\epsilon), \cdots, \lambda_{m}(\epsilon)\right)$, where the $l$ first elements are negative and the $m-l$ last elements are positive. $y=\left(\begin{array}{l}y^{-} \\ y^{+}\end{array}\right)$where $y^{-}:[0,1] \times[0,+\infty) \rightarrow \mathbb{R}^{i}$ and $y^{+}:[0,1] \times$ $[0,+\infty) \rightarrow \mathbb{R}^{n-i} . z=\left(\begin{array}{c}z^{-} \\ z^{+}\end{array}\right)$where $z^{-}:[0,1] \times[0,+\infty) \rightarrow$ $\mathbb{R}^{l}$ and $z^{+}:[0,1] \times[0,+\infty) \rightarrow \mathbb{R}^{m-l} .0<\epsilon \ll 1$. The matrices $a(\epsilon), b(\epsilon), c(\epsilon)$ and $d(\epsilon)$ are in appropriate dimensions and vanish at $\epsilon=0$.

The boundary condition under consideration is given by

$$
\left(\begin{array}{l}
y^{-}(1, t) \\
y^{+}(0, t) \\
z^{-}(1, t) \\
z^{+}(0, t)
\end{array}\right)=G(\epsilon)\left(\begin{array}{c}
y^{-}(0, t) \\
y^{+}(1, t) \\
z^{-}(0, t) \\
z^{+}(1, t)
\end{array}\right), \quad t \in[0,+\infty)
$$


where $G(\epsilon)=\left(\begin{array}{ll}G_{11}(\epsilon) & G_{12}(\epsilon) \\ G_{21}(\epsilon) & G_{22}(\epsilon)\end{array}\right)$ is a matrix in $\mathbb{R}^{(n+m) \times(n+m)}$ with the matrices $G_{11}(\epsilon)$ in $\mathbb{R}^{n \times n}, G_{12}(\epsilon)$ in $\mathbb{R}^{n \times m}, G_{21}(\epsilon)$ in $\mathbb{R}^{m \times n}, G_{22}(\epsilon)$ in $\mathbb{R}^{m \times m}$. Given two functions $y^{0}:[0,1] \rightarrow$ $\mathbb{R}^{n}$ and $z^{0}:[0,1] \rightarrow \mathbb{R}^{m}$, the initial condition is

$$
\left(\begin{array}{l}
y(x, 0) \\
z(x, 0)
\end{array}\right)=\left(\begin{array}{c}
y^{0}(x) \\
z^{0}(x)
\end{array}\right), \quad x \in[0,1] .
$$

Replacing $y(x, t)$ by $\left(\begin{array}{c}y^{-}(1-x, t) \\ y^{+}(x, t)\end{array}\right)$ and $z(x, t)$ by $\left(\begin{array}{c}z^{-}(1-x, t) \\ z^{+}(x, t)\end{array}\right)$, it may be assumed, without loss of generality, that the matrices $\Lambda_{1}(\epsilon)$ and $\Lambda_{2}(\epsilon)$ are diagonal positive. The full system (1) can then be rewritten under the form

$$
\begin{aligned}
y_{t}(x, t)+\Lambda_{1}(\epsilon) y_{x}(x, t) & =a^{+}(\epsilon) y(x, t)+a^{-}(\epsilon) y(1-x, t) \\
& +b^{+}(\epsilon) z(x, t)+b^{-}(\epsilon) z(1-x, t),
\end{aligned}
$$

$$
\begin{aligned}
\epsilon z_{t}(x, t)+\Lambda_{2}(\epsilon) z_{x}(x, t) & =c^{+}(\epsilon) y(x, t)+c^{-}(\epsilon) y(1-x, t) \\
& +d^{+}(\epsilon) z(x, t)+d^{-}(\epsilon) z(1-x, t) .
\end{aligned}
$$

Then the boundary condition (2) becomes

$$
\left(\begin{array}{c}
y(0, t) \\
z(0, t)
\end{array}\right)=G(\epsilon)\left(\begin{array}{c}
y(1, t) \\
z(1, t)
\end{array}\right), \quad t \in[0,+\infty) .
$$

Adapting the approach in [13], [8] to infinite dimensional systems, the reduced subsystem for (4) and (5) is formally computed as follows. By setting $\epsilon=0$ in (4b), we get $z_{x}(x, t)=0$, which implies $z(., t)=z(1, t)$. Substituting it into the second line of the boundary condition (5) and assuming $\left(I_{m}-G_{22}(0)\right)$ invertible, we obtain $z(., t)=\left(I_{m}-\right.$ $\left.G_{22}(0)\right)^{-1} G_{21}(0) y(1, t)$ and $y(0, t)=\left(G_{11}(0)+G_{12}(0)\left(I_{m}-\right.\right.$ $\left.\left.G_{22}(0)\right)^{-1} G_{21}(0)\right) y(1, t)$. The reduced subsystem is thus written as

$$
\bar{y}_{t}(x, t)+\Lambda_{1}(0) \bar{y}_{x}(x, t)=0, \quad x \in[0,1], \quad t \in[0,+\infty),
$$

with the boundary condition

$$
\bar{y}(0, t)=G_{r} \bar{y}(1, t), \quad t \in[0,+\infty),
$$

where $G_{r}=G_{11}(0)+G_{12}(0)\left(I_{m}-G_{22}(0)\right)^{-1} G_{21}(0)$, whereas the initial condition is given as the same as for the full system

$$
\bar{y}(x, 0)=\bar{y}^{0}(x)=y^{0}(x), \quad x \in[0,1] .
$$

The compatibility conditions for the existence of solutions of (6)-(8) in $H^{2}$-norm are given as follows

$$
\begin{aligned}
& \bar{y}^{0}(0)=G_{r} \bar{y}^{0}(1), \\
& \bar{y}_{x}^{0}(0)=\Lambda_{1}^{-1}(0) G_{r} \Lambda_{1}(0) \bar{y}_{x}^{0}(1) .
\end{aligned}
$$

Remark 1. Compared with [19], the transport velocities of the full system in the present work depend on $\epsilon$ as well as the boundary conditions. Moreover, we consider an additional source term which is also dependent on $\epsilon$. Due to the presence of $\epsilon$ in both dynamics and boundary conditions, the full system becomes more complex. The assumptions on the continuity for such terms with respect to $\epsilon$ should be used to ensure that the Tikhonov approximation is valid for $\epsilon$ sufficiently small. The proof of the main result is then more sophisticated and is a non trivial extension.

\section{TIKHONOV APPROXIMATION OF LINEAR HYPERBOLIC SYSTEMS OF BALANCE LAWS}

In this section, the approximation of the solutions to the full system by that to the reduced subsystem is established by Lyapunov techniques. First let us consider the following assumptions.

Assumption 1. The functions $\Lambda_{1}$ and $\Lambda_{2}$ are Lipschitz continuous at 0 , that is there exist positive constants $R_{1}$ and $\bar{\epsilon}$ such that for all $0<\epsilon<\bar{\epsilon}$,

$$
\left\|\Lambda_{1}(\epsilon)-\Lambda_{1}(0)\right\| \leqslant \epsilon R_{1},\left\|\Lambda_{2}(\epsilon)-\Lambda_{2}(0)\right\| \leqslant \epsilon R_{1} .
$$

Assumption 2. Let $\bar{\epsilon}$ as in Assumption 1, the functions $a, b$, $c$ and $d$ are Lipschitz continuous at 0 , that is there exits a positive constant $R_{2}$, such that for all $0<\epsilon<\bar{\epsilon}$,

$\|a(\epsilon)\| \leqslant \epsilon R_{2},\|b(\epsilon)\| \leqslant \epsilon R_{2},\|c(\epsilon)\| \leqslant \epsilon R_{2},\|d(\epsilon)\| \leqslant \epsilon R_{2}$.

Assumption 3. Let $\bar{\epsilon}$ as in Assumption 1, the functions $G_{11}$, $G_{12}, G_{21}$ and $G_{22}$ are Lipschitz continuous at 0 , that is there exists a positive value $R_{3}$, such that for all $0<\epsilon<\bar{\epsilon}$,

$\left\|G_{11}(\epsilon)-G_{11}(0)\right\| \leqslant \epsilon R_{3}, \quad\left\|G_{12}(\epsilon)-G_{12}(0)\right\| \leqslant \epsilon R_{3}$, $\left\|G_{21}(\epsilon)-G_{21}(0)\right\| \leqslant \epsilon R_{3}, \quad\left\|G_{22}(\epsilon)-G_{22}(0)\right\| \leqslant \epsilon R_{3}$.

We are ready to state the main result in the following theorem. Theorem 1. Consider the linear hyperbolic system (4)-(5), under Assumptions 1-3, if $\rho_{1}(G(0))<1$, there exist positive values $C_{1}, C_{2}, \theta, \epsilon^{*}$ such that for all $0<\epsilon<\epsilon^{*}$, for any initial condition $y^{0} \in H^{2}(0,1)$ satisfying compatibility conditions (9) with $\bar{y}^{0}=y^{0}$, and $z^{0} \in L^{2}(0,1)$, it holds for all $t \geqslant 0$

$$
\begin{aligned}
& \|y(., t)-\bar{y}(., t)\|_{L^{2}}^{2} \leqslant \epsilon C_{1} e^{-\theta t}\left(\left\|\bar{y}^{0}\right\|_{H^{2}}^{2}\right. \\
& \left.+\left\|z^{0}-\left(I_{m}-G_{22}(0)\right)^{-1} G_{21}(0) \bar{y}^{0}(1)\right\|_{L^{2}}^{2}\right)
\end{aligned}
$$

$$
\begin{aligned}
& \int_{0}^{+\infty}\left\|z(., t)-\left(I_{m}-G_{22}(0)\right)^{-1} G_{21}(0) \bar{y}(1, t)\right\|_{L^{2}}^{2} d t \leqslant \\
& \epsilon C_{2}\left(\left\|\bar{y}^{0}\right\|_{H^{2}}^{2}+\left\|z^{0}-\left(I_{m}-G_{22}(0)\right)^{-1} G_{21}(0) \bar{y}^{0}(1)\right\|_{L^{2}}^{2}\right) .
\end{aligned}
$$

Corollary 1. If $\rho_{1}(G(0))<1$, under Assumptions 1-3, the full system (4) with the boundary condition (5) is exponentially stable in $L^{2}$-norm for all $0<\epsilon<\epsilon^{*}$.

The proofs of Theorem 1 and Corollary 1 are given in the following section.

\section{PRoOf of Theorem 1 AND COROLlary 1}

Proof of Theorem 1: In the following we will use three steps to prove Theorem 1 .

Step 1) Let us perform the following change of variables,

$$
\begin{aligned}
& \eta(x, t)=y(x, t)-\bar{y}(x, t), \\
& \delta(x, t)=z(x, t)-\left(I_{m}-G_{22}(0)\right)^{-1} G_{21}(0) \bar{y}(1, t),
\end{aligned}
$$

where $\eta$ stands for the error between the slow dynamics $y$ in (4) and $\bar{y}$ in (6), and $\delta$ is the error between the fast dynamics 
$z$ in (4) and its equilibrium point. In all the following, it is assumed $\epsilon \in(0, \bar{\epsilon})$. Due to (10) and (6), the system (4) can be rewritten in the new variables $(\eta, \delta)$ as follows

$$
\begin{aligned}
& \eta_{t}(x, t)+\Lambda_{1}(\epsilon) \eta_{x}(x, t)=a^{+}(\epsilon) \eta(x, t)+a^{-}(\epsilon) \eta(1-x, t) \\
& \quad+b^{+}(\epsilon) \delta(x, t)+b^{-}(\epsilon) \delta(1-x, t)+a^{+}(\epsilon) \bar{y}(x, t) \\
& \quad+a^{-}(\epsilon) \bar{y}(1-x, t)-\left(\Lambda_{1}(\epsilon)-\Lambda_{1}(0)\right) \bar{y}_{x}(x, t) \\
& \quad+b(\epsilon)\left(I_{m}-G_{22}(0)\right)^{-1} G_{21}(0) \bar{y}(1, t), \\
& \epsilon \delta_{t}(x, t)+\Lambda_{2}(\epsilon) \delta_{x}(x, t)=c^{+}(\epsilon) \eta(x, t)+c^{-}(\epsilon) \eta(1-x, t) \\
& \quad+d^{+}(\epsilon) \delta(x, t)+d^{-}(\epsilon) \delta(1-x, t) \\
& \quad+c^{+}(\epsilon) \bar{y}(x, t)+c^{-}(\epsilon) \bar{y}(1-x, t) \\
& \quad+d(\epsilon)\left(I_{m}-G_{22}(0)\right)^{-1} G_{21}(0) \bar{y}(1, t) \\
& \quad+\epsilon\left(I_{m}-G_{22}(0)\right)^{-1} G_{21}(0) \Lambda_{1}(0) \bar{y}_{x}(1, t) .
\end{aligned}
$$

Due to (5) and (7), the boundary condition for system (11) is computed as follows

$$
\left(\begin{array}{l}
\eta(0, t) \\
\delta(0, t)
\end{array}\right)=\left(\begin{array}{ll}
G_{11}(\epsilon) & G_{12}(\epsilon) \\
G_{21}(\epsilon) & G_{22}(\epsilon)
\end{array}\right)\left(\begin{array}{l}
\eta(1, t) \\
\delta(1, t)
\end{array}\right)+\left(\begin{array}{l}
G_{11}(\epsilon) \\
G_{d 2}(\epsilon)
\end{array}\right) \bar{y}(1, t),
$$

where $G_{d 1}(\epsilon)=\left(G_{11}(\epsilon)-G_{11}(0)\right)+\left(G_{12}(\epsilon)-G_{12}(0)\right)\left(I_{m}-\right.$ $\left.G_{22}(0)\right)^{-1} G_{21}(0)$ and $G_{d 2}(\epsilon)=\left(G_{21}(\epsilon)-G_{21}(0)\right)+$ $\left(G_{22}(\epsilon)-G_{22}(0)\right)\left(I_{m}-G_{22}(0)\right)^{-1} G_{21}(0)$.

Remark 2. Due to Assumption 3, there exists a positive constant $r_{1}$, such that $\left\|G_{d 1}(\epsilon)\right\| \leqslant \epsilon r_{1},\left\|G_{d 2}(\epsilon)\right\| \leqslant \epsilon r_{1}$. $\quad$ ○

The candidate Lyapunov function for system (11)-(12) is $V=V_{1}+V_{2}$ with $V_{1}=\int_{0}^{1} e^{-\mu x} \eta^{\top}(x, t) Q \eta(x, t) d x$ and $V_{2}=\epsilon \int_{0}^{1} e^{-\mu x} \delta^{\top}(x, t) P \delta(x, t) \quad d x$, where $\mu>$ $0, Q$ a positive diagonal matrix in $\mathbb{R}^{n \times n}$ and $P$ a positive diagonal matrix in $\mathbb{R}^{m \times m}$. Let us compute the time derivative of $V_{1}$ along (11a), we get $\dot{V}_{1}=$ $\int_{0}^{1} e^{-\mu x}\left(2 \eta^{\top}(x, t) Q \eta_{t}(x, t)\right) \quad d x$. Using the expression in (11a) to replace $\eta_{t}(x, t)$ and performing an integration by parts for the integral $2 \int_{0}^{1} e^{-\mu x} \eta^{\top}(x, t) Q \Lambda_{1}(\epsilon) \eta_{x}(x, t) d x$ yield

$\dot{V}_{1}=-\left[e^{-\mu x} \eta^{\top}(x) Q \Lambda_{1}(\epsilon) \eta(x)\right]_{x=0}^{x=1}$

$-\int_{0}^{1} e^{-\mu x} \eta^{\top}(x, t)\left(\mu Q \Lambda_{1}(\epsilon)-2 Q a^{+}(\epsilon)\right) \eta(x, t) d x$

$+2 \int_{0}^{1} e^{-\mu x} \eta^{\top}(x, t) Q a^{-}(\epsilon) \eta(1-x, t) d x$

$+2 \int_{0}^{1} e^{-\mu x} \eta^{\top}(x, t) Q b^{+}(\epsilon) \delta(x, t) d x$

$+2 \int_{0}^{1} e^{-\mu x} \eta^{\top}(x, t) Q b^{-}(\epsilon) \delta(1-x, t) d x$

$+2 \int_{0}^{1} e^{-\mu x} \eta^{\top}(x, t) Q a^{+}(\epsilon) \bar{y}(x, t) d x$

$+2 \int_{0}^{1} e^{-\mu x} \eta^{\top}(x, t) Q a^{-}(\epsilon) \bar{y}(1-x, t) d x$

$-2 \int_{0}^{1} e^{-\mu x} \eta^{\top}(x, t) Q\left(\Lambda_{1}(\epsilon)-\Lambda_{1}(0)\right) \bar{y}_{x}(x, t) d x$

$+2 \int_{0}^{1} e^{-\mu x} \eta^{\top}(x, t) Q b(\epsilon)\left(I_{m}-G_{22}(0)\right)^{-1} G_{21}(0) \bar{y}(1, t) d x$.

Similarly, we compute the time derivative of $V_{2}$ along (11b) yield

$\dot{V}_{2}=-\left[e^{-\mu x} \delta^{\top}(x) P \Lambda_{2}(\epsilon) \delta(x)\right]_{x=0}^{x=1}$

$-\int_{0}^{1} e^{-\mu x} \delta^{\top}(x, t)\left(\mu P \Lambda_{2}(\epsilon)-2 P d^{+}(\epsilon)\right) \delta(x, t) d x$

$+2 \int_{0}^{1} e^{-\mu x} \delta^{\top}(x, t) P d^{-}(\epsilon) \delta(1-x, t) d x$

$+2 \int_{0}^{1} e^{-\mu x} \delta^{\top}(x, t) P c^{+}(\epsilon) \eta(x, t) d x$

$+2 \int_{0}^{1} e^{-\mu x} \delta^{\top}(x, t) P c^{-}(\epsilon) \eta(1-x, t) d x$

$+2 \int_{0}^{1} e^{-\mu x} \delta^{\top}(x, t) P c^{+}(\epsilon) \bar{y}(x, t) d x$

$+2 \int_{0}^{1} e^{-\mu x} \delta^{\top}(x, t) P c^{-}(\epsilon) \bar{y}(1-x, t) d x$

$+2 \int_{0}^{1} e^{-\mu x} \delta^{\top} P d(\epsilon)\left(I_{m}-G_{22}(0)\right)^{-1} G_{21}(0) \bar{y}(1, t) d x$

$+2 \epsilon \int_{0}^{1} e^{-\mu x} \delta^{\top} P\left(I_{m}-G_{22}(0)\right)^{-1} G_{21}(0) \Lambda_{1}(0) \bar{y}_{x}(1, t) d x$.

Combining $\dot{V}_{1}$ and $\dot{V}_{2}$, we obtain $\dot{V}(\eta, \delta, \epsilon)=\dot{V}_{1}+\dot{V}_{2}=$ $T_{1}+T_{2}+T_{3}$, with:

$$
\begin{aligned}
& T_{1}=- {\left[e^{-\mu x}\left(\eta^{\top}(x) Q \Lambda_{1}(\epsilon) \eta(x)+\delta^{\top}(x) P \Lambda_{2}(\epsilon) \delta(x)\right)\right]_{x=0}^{x=1} } \\
& T_{2}=-\int_{0}^{1} e^{-\mu x} \eta^{\top}(x, t)\left(\mu Q \Lambda_{1}(\epsilon)-2 Q a^{+}(\epsilon)\right) \eta(x, t) d x \\
&-\int_{0}^{1} e^{-\mu x} \delta^{\top}(x, t)\left(\mu P \Lambda_{2}(\epsilon)-2 P d^{+}(\epsilon)\right) \delta(x, t) d x \\
&+2 \int_{0}^{1} e^{-\mu x} \eta^{\top}(x, t)\left(Q b^{+}(\epsilon)+c^{+\top}(\epsilon) P\right) \delta(x, t) d x \\
&+2 \int_{0}^{1} e^{-\mu x} \eta^{\top}(x, t) Q a^{-}(\epsilon) \eta(1-x, t) d x \\
&+2 \int_{0}^{1} e^{-\mu x} \eta^{\top}(x, t) Q b^{-}(\epsilon) \delta(1-x, t) d x \\
&+2 \int_{0}^{1} e^{-\mu x} \delta^{\top}(x, t) P d^{-}(\epsilon) \delta(1-x, t) d x \\
&+2 \int_{0}^{1} e^{-\mu x} \delta^{\top}(x, t) P c^{-}(\epsilon) \eta(1-x, t) d x \\
&+2 \int_{0}^{1} e^{-\mu x} \delta^{\top}(x, t) P c^{-}(\epsilon) \bar{y}(1-x, t) d x \\
&+2 \int_{0}^{1} e^{-\mu x} \eta^{\top}(x, t) Q b(\epsilon)\left(I_{m}-G_{22}(0)\right)^{-1} G_{21}(0) \bar{y}(1, t) d x \\
&+2 \int_{0}^{1} e^{-\mu x} \eta^{\top}(x, t) Q a^{+}(\epsilon) \bar{y}(x, t) d x \\
&+2 \int_{0}^{1} e^{-\mu x} \delta^{\top}(x, t) P c^{+}(\epsilon) \bar{y}(x, t) d x \\
&+\mu x
\end{aligned}
$$


$+2 \int_{0}^{1} e^{-\mu x} \delta^{\top}(x, t) P d(\epsilon)\left(I_{m}-G_{22}(0)\right)^{-1} G_{21}(0) \bar{y}(1, t) d x$ $+2 \epsilon \int_{0}^{1} e^{-\mu x} \delta^{\top}(x, t) P\left(I_{m}-G_{22}(0)\right)^{-1} G_{21}(0) \Lambda_{1}(0) \bar{y}_{x}(1, t) d x$.

Step 2) To estimate the terms $T_{1}-T_{3}$, let us state the following lemmas. The stability of the reduced subsystem in $H^{2}$-norm is given in Lemma 1.

Lemma 1. [19] Consider the reduced subsystem (6)-(8), if $\rho_{1}(G(0))<1$, there exist $C_{r}>0$, such that for any initial condition $\bar{y}^{0} \in H^{2}(0,1)$ satisfying the compatibility conditions (9) and for all $t \geqslant 0$,

$$
\|\bar{y}(., t)\|_{H^{2}}^{2} \leqslant C_{r} e^{-\mu \underline{\lambda}\left(\Lambda_{1}(0)\right) t}\left\|\bar{y}^{0}\right\|_{H^{2}}^{2} .
$$

Lemma 2. If $\rho_{1}(G(0))<1$, under Assumptions 1 and 3, there exist positive values $C_{T_{1}}$ and $\epsilon_{1}^{*}$, such that for all $\epsilon \in\left(0, \epsilon_{1}^{*}\right)$ and $t \geqslant 0$,

$$
T_{1} \leqslant \epsilon C_{T_{1}} e^{-\mu \underline{\lambda}\left(\Lambda_{1}(0)\right) t}\left\|\bar{y}^{0}\right\|_{H^{2}}^{2} .
$$

Lemma 3. Under Assumptions 1 and 2, there exist positive values $C_{T_{2}}$ and $\epsilon_{2}^{*}$, such that for all $\epsilon \in\left(0, \epsilon_{2}^{*}\right)$,

$$
T_{2} \leqslant-C_{T_{2}} \int_{0}^{1} e^{-\mu x}\left(\eta^{\top} Q \eta+\delta^{\top} P \delta\right) d x .
$$

Lemma 4. Under Assumptions 1 and 2, there exist positive constants $C_{T_{31}}, C_{T_{32}}$ and $C_{T_{33}}$, such that for all positive value $\epsilon$ and for all $t \geqslant 0$,

$$
\begin{aligned}
T_{3} \leqslant & \epsilon C_{T_{31}} \int_{0}^{1} e^{-\mu x}|\eta|^{2} d x+\epsilon C_{T_{32}} \int_{0}^{1} e^{-\mu x}|\delta|^{2} d x \\
& +\epsilon C_{T_{33}} e^{-\mu \underline{\lambda}\left(\Lambda_{1}(0)\right) t}\left\|\bar{y}^{0}\right\|_{H^{2}}^{2}
\end{aligned}
$$

The proofs of Lemmas 2-4 are collected in [17].

Remark 3. The choice of matrices $P, Q$ and positive value $\mu$ is constrained in the proof of Lemma 2 to satisfy a matrix inequality which is solvable as soon as $\rho_{1}(G(0))<1$.

Step 3) Using Lemmas 2-4, we obtain

$$
\begin{aligned}
\dot{V}(\eta, \delta, \epsilon) \leqslant & -\left(C_{T_{2}}-\epsilon C_{v}\right) \int_{0}^{1} e^{-\mu x}\left(\eta^{\top} Q \eta+\delta^{\top} P \delta\right) d x \\
& +\epsilon\left(C_{T_{1}}+C_{T_{33}}\right) e^{-\mu \underline{\lambda}\left(\Lambda_{1}(0)\right) t}\left\|\bar{y}^{0}\right\|_{H^{2}}^{2},
\end{aligned}
$$

where $C_{v}=\max \left(\frac{C_{T_{31}}}{\underline{\lambda}(Q)}, \frac{C_{T_{32}}}{\underline{\lambda}(P)}\right)$. Let $\epsilon_{3}^{*}=\frac{C_{T_{2}}}{2 C_{v}}, \epsilon_{1}^{*}$ in Lemma $2, \epsilon_{2}^{*}$ in Lemma 3 and $\epsilon^{*}=\min \left(\epsilon_{1}^{*}, \epsilon_{2}^{*}, \epsilon_{3}^{*}\right)$, there exists $\varpi>0$ such that for all $\epsilon \in\left(0, \epsilon^{*}\right)$,

$\dot{V}(\eta, \delta, \epsilon) \leqslant-\varpi V(\eta, \delta, \epsilon)+\epsilon\left(C_{T_{1}}+C_{T_{33}}\right) e^{-\mu \underline{\lambda}\left(\Lambda_{1}(0)\right) t}\left\|\bar{y}^{0}\right\|_{H^{2}}^{2}$.

In the above inequality, the term $\left\|\bar{y}^{0}\right\|_{H^{2}}^{2}$ is seen as a disturbance and it follows that

$$
\begin{aligned}
& V(\eta, \delta, \epsilon) \leqslant e^{-\varpi t} V\left(\eta^{0}, \delta^{0}, \epsilon\right) \\
& +\epsilon\left(C_{T_{1}}+C_{T_{33}}\right) e^{-\varpi t} \frac{e^{\left(\varpi-\mu \underline{\lambda}\left(\Lambda_{1}(0)\right) t\right.}-1}{\varpi-\mu \underline{\lambda}\left(\Lambda_{1}(0)\right)}\left\|\bar{y}^{0}\right\|_{H^{2}}^{2} .
\end{aligned}
$$

Since $\varpi<C_{T_{2}}$, we may let $\varpi<\mu \underline{\lambda}\left(\Lambda_{1}(0)\right)$, thus (18) can be rewritten as follows $V(\eta, \delta, \epsilon) \leqslant$ $e^{-\varpi t} V\left(\eta^{0}, \delta^{0}, \epsilon\right)+\epsilon \bar{M} e^{-\varpi t}\left\|\bar{y}^{0}\right\|_{H^{2}}^{2}$. Since $V(\eta, \delta, \epsilon)$ is lower and upper estimated by $e^{-\mu} \underline{\lambda}(Q)\|\eta\|_{L^{2}}^{2}+\epsilon e^{-\mu} \underline{\lambda}(P)\|\delta\|_{L^{2}}^{2} \leqslant$
$V(\eta, \delta, \epsilon) \leqslant\|Q\|\|\eta\|_{L^{2}}^{2}+\epsilon\|P\|\|\delta\|_{L^{2}}^{2}$, it follows $\|\eta(., t)\|_{L^{2}}^{2} \leqslant$ $\frac{e^{\mu} e^{-\varpi t}}{\underline{\lambda}(Q)} V\left(\eta^{0}, \delta^{0}, \epsilon\right)+\frac{\epsilon \bar{M} e^{\mu} e^{-\varpi t}}{\underline{\lambda}(Q)}\left\|\bar{y}^{0}\right\|_{H^{2}}^{2}$. Due to the initial condition $y^{0}=\bar{y}^{0}$ i.e. $\eta^{0} \stackrel{\underline{\lambda}}{=} 0$, the following inequality holds

$\|\eta(., t)\|_{L^{2}}^{2} \leqslant \frac{\epsilon\|P\| e^{\mu} e^{-\varpi t}}{\underline{\lambda}(Q)}\left\|\delta^{0}\right\|_{L^{2}}+\frac{\epsilon \bar{M} e^{\mu} e^{-\varpi t}}{\underline{\lambda}(Q)}\left\|\bar{y}^{0}\right\|_{H^{2}}^{2}$.

This proves the first inequality in Theorem 1 .

Noting that for $\epsilon<\epsilon^{*}$, the term $-\left(C_{T_{2}}-\right.$ $\left.\epsilon C_{v}\right) \int_{0}^{1} e^{-\mu x} \eta^{\top} Q \eta d x$ in the right hand side of (17) is always negative, then $\dot{V}(\eta, \delta, \epsilon)$ is rewritten as follows $\dot{V}(\eta, \delta, \epsilon) \leqslant$ $-\varpi \int_{0}^{1} e^{-\mu x} \delta^{\top} P \delta d x+\epsilon\left(C_{T_{1}}+C_{T_{33}}\right) e^{-\mu \underline{\lambda}\left(\Lambda_{1}(0)\right) t}\left\|\bar{y}^{0}\right\|_{H^{2}}^{2}$. Performing an integration of both sides from 0 to $+\infty$, it follows

$$
\begin{array}{r}
\int_{0}^{+\infty}\|\delta(., t)\|_{L^{2}}^{2} d t \leqslant \frac{e^{\mu}}{\underline{\lambda}(P) \varpi}\left(V\left(\eta^{0}, \delta^{0}, \epsilon\right)-\lim _{t \rightarrow+\infty} V(\eta, \delta, \epsilon)\right. \\
\left.+\epsilon\left(C_{T_{1}}+C_{T_{33}}\right)\left\|\bar{y}^{0}\right\|_{H^{2}}^{2} \int_{0}^{+\infty} e^{-\mu \underline{\lambda}\left(\Lambda_{1}(0)\right) t} d t\right)
\end{array}
$$

since $\lim _{t \rightarrow+\infty} V(\eta, \delta, \epsilon)=0$ and $\eta^{0}=0$, it follows

$\int_{0}^{+\infty}\|\delta(., t)\|_{L^{2}}^{2} d t \leqslant \frac{\epsilon e^{\mu}\|P\|}{\underline{\lambda}(P) \varpi}\left\|\delta^{0}\right\|_{L^{2}}+\frac{\epsilon e^{\mu}\left(C_{T_{1}}+C_{T_{33}}\right)}{\mu \underline{\lambda}(P) \underline{\lambda}\left(\Lambda_{1}(0)\right) \varpi}\left\|\bar{y}^{0}\right\|_{H^{2}}^{2}$.

This proves the second inequality in Theorem 1 and concludes the proof of this theorem.

Proof of Corollary 1: Due to (13), the reduced subsystem is exponentially stable in $H^{2}$-norm. The error system (11)-(12) is exponentially stable in $L^{2}$-norm according to (18). By (10) we prove that the full system is exponentially stable in $L^{2}$-norm.

\section{NumERICAL RESUlTS}

\section{A. Academic example}

We consider the following academic example which illustrates the full generality of our result. Consider system (4) with $\Lambda_{1}(\epsilon)=1+\epsilon, \Lambda_{2}(\epsilon)=\epsilon-1, a(\epsilon)=0.1 \epsilon$, $b(\epsilon)=0.2 \epsilon, c(\epsilon)=0.05 \epsilon$ and $d(\epsilon)=0.4 \epsilon$, which satisfies Assumptions 1 and 2. The boundary condition (5) is given by $G(\epsilon)=\left(\begin{array}{cc}0.5+\epsilon & 1+\epsilon \\ 0.5+\epsilon & -0.5+\epsilon\end{array}\right)$, thus Assumption 3 holds. Considering a diagonal positive matrix $\Delta=\left(\begin{array}{cc}0.5 & 0 \\ 0 & 0.7\end{array}\right)$, it holds $\left\|\Delta G(0) \Delta^{-1}\right\|<1$. Thus $\rho_{1}(G(0))<1$ is satisfied. Theorem 1 applies. To numerically compute the solutions of this example, we discretize it by using a two-step variant of the Lax-Wendroff method (see [14] and [15]). Precisely, the space domain $[0,1]$ is divided into 100 intervals of identical length, the final time is chosen as 30 . We take a time-step $d t=$ $(0.9 \epsilon /|\epsilon-1|) d x$ that satisfies the CFL condition and select the initial conditions $y^{0}(x)=1-\cos (4 \pi x), z^{0}(x)=\sin (2 \pi x)$, for all $x \in[0,1]$, such that the compatibility condition is satisfied. The evolutions of $\|\eta(., t=3)\|_{L^{2}}^{2}$ and of $\int_{0}^{30}\|\delta(., t)\|_{L^{2}}^{2} d t$ for different $\epsilon$ are given by Table I. The values are close to zero and decrease as $\epsilon$ decreases. 


\begin{tabular}{|c|c|c|c|}
\hline$\epsilon$ & 0.005 & 0.01 & 0.015 \\
\hline$\|\eta(., t=3)\|_{L^{2}}^{2}$ & $3 \times 10^{-3}$ & $1.2 \times 10^{-2}$ & $2.8 \times 10^{-2}$ \\
\hline $\int_{0}^{30}\|\delta(., t)\|_{L^{2}}^{2} d t$ & $7 \times 10^{-3}$ & $2.6 \times 10^{-2}$ & $5.7 \times 10^{-2}$ \\
\hline
\end{tabular}

TABLE I: Evolutions of square of $L^{2}$-norm of $\eta$ and of time integral of square of $L^{2}$-norm of $\delta$ for different $\epsilon$

Remark 4. The simulation cost is lower when we simulate the reduced subsystem with a time-step which does not depend on $\epsilon$ and satisfies the CFL condition $\bar{\lambda}\left(\Lambda_{1}(0)\right) d t<d x$ than simulating the full system by using a smaller time-step satisfying CFL condition $\bar{\lambda}\left(\Lambda_{2}(\epsilon)\right) d t<\epsilon d x$.

\section{B. Physical application}

a) System description: The gas dynamics through a constant cross section tube, where all the friction losses and heat transfers are neglected, can be modeled by the following Euler equations as considered in [20, Chapter 2], by considering a tube of length equals to 1 .

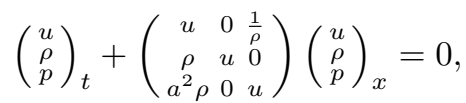

where $u=u(x, t)$ stands for the gas velocity at location $x$ in $[0,1]$ and at time $t ; \rho=\rho(x, t)$ represents the gas density; $p=p(x, t)$ is the gas pressure; $a$ is sound speed in ideal gas. System (19) admits a constant in space steadystate $\left(u^{*}, \rho^{*}, p^{*}\right)$. The deviations of the state $(u, \rho, p)$ around the steady-state are defined as $\bar{u}=u-u^{*}, \bar{\rho}=\rho-\rho^{*}$, $\bar{p}=p-p^{*}$. Then the linearization of system (19) at this equilibrium is given by

$$
\left(\begin{array}{l}
\bar{u} \\
\bar{\rho}
\end{array}\right)_{t}+\left(\begin{array}{ccc}
u^{*} & 0 & \frac{1}{\rho^{*}} \\
\rho^{*} & u^{*} & 0 \\
a^{* 2} \rho^{*} & 0 & u^{*}
\end{array}\right)\left(\begin{array}{l}
\bar{u} \\
\bar{\rho}
\end{array}\right)_{x}=0
$$

Performing a change of variable in Riemann coordinates and assuming that the propagation speed of gas is much slower than the sound speed, i.e. $u<<a$, we define $\epsilon=\frac{u^{*}}{a^{*}}$, then (20) can be written as a singularly perturbed system

$$
\left(\begin{array}{c}
M_{1} \\
\epsilon M_{2} \\
\epsilon M_{3}
\end{array}\right)_{t}+\left(\begin{array}{ccc}
u^{*} & 0 & 0 \\
0 & u^{*}(\epsilon-1) & 0 \\
0 & 0 & u^{*}(1+\epsilon)
\end{array}\right)\left(\begin{array}{l}
M_{1} \\
M_{2} \\
M_{3}
\end{array}\right)_{x}=0
$$

with $M=\left(\begin{array}{c}M_{1} \\ M_{2} \\ M_{3}\end{array}\right)=\left(\begin{array}{ccc}0 & 1 & 1 \\ 1 & -\frac{\rho^{*}}{a^{*}} & \frac{\rho^{*}}{a^{*}} \\ 0 & -a^{*} \rho^{*} & a^{*} \rho^{*}\end{array}\right)^{-1}\left(\begin{array}{c}\bar{u} \\ \bar{\rho}\end{array}\right)$.

b) Boundary conditions: The setup is provided with fans which are located at the two extremities of the tube. The rotation speed is considered as the control action. We consider the following three boundary conditions for system (19).

1. The first boundary condition describes the operation of the inflow fan (see the fan specification map in [21]),

$$
u(0, t) s=\sigma c_{0}(t)\left(p(0, t)-p_{i n}\right),
$$

where $s$ stands for the tube's constant cross section, $\sigma$ is a constant coefficient, the control input is denoted by $c_{0}(t)$ and $p_{i n}$ is a constant pressure before the inflow fan.

2 . Similarly, the second boundary condition is given by the outflow fan,

$$
u(1, t) s=\sigma c_{1}(t)\left(p_{\text {out }}-p(1, t)\right),
$$

the control input is denoted by $c_{1}(t)$ and $p_{\text {out }}$ is a constant pressure behind the outflow fan.

3 . The third boundary condition is a physical constraint. Precisely, the gas pressure at the inflow fan is close to the atmospheric pressure (see [2]),

$$
\rho(0, t)=\tilde{\rho}
$$

where $\tilde{\rho}$ is constant.

The boundary conditions for system (20) are obtained by linearizing the above three boundary conditions,

$$
\begin{aligned}
\bar{u}(0, t) s & =\sigma\left[\bar{c}_{0}(t)\left(p^{*}-p_{\text {in }}\right)+c_{0}^{*} \bar{p}(0, t)\right], \\
\bar{u}(1, t) s & =\sigma\left[\bar{c}_{1}(t)\left(p_{\text {out }}-p^{*}\right)-c_{1}^{*} \bar{p}(1, t)\right], \\
\bar{\rho}(0, t) & =0,
\end{aligned}
$$

where $c_{0}^{*}, c_{1}^{*}$ are the constant control actions at the steady-state $\left(u^{*}, \rho^{*}, p^{*}\right)$.

Proposition 1. For any values $K_{23}$ and $K_{32}$ in $\mathbb{R}$, such that $K_{23} \neq 1$ and $K_{32} \neq 1$, defining control actions by

$c_{0}(t)=c_{0}^{*}+\frac{\frac{s\left(1+K_{32}\right)}{\sigma a^{*} \rho^{*}\left(K_{32}-1\right)}-c_{0}^{*}}{p^{*}-p_{i n}} \bar{p}(0, t)$,

$c_{1}(t)=c_{1}^{*}+\frac{\frac{s\left(a^{*}\left(1+K_{23}\right)-2 \rho^{*} K_{21}\right)}{\sigma a^{* 2} \rho^{*}\left(1-K_{23}\right)}+c_{1}^{*}}{p_{\text {out }}-p^{*}} \bar{p}(1, t)+\frac{\frac{2 s K_{21}}{\sigma\left(1-K_{23}\right)}}{p_{\text {out }}-p^{*}} \bar{\rho}(1, t)$,

the following conditions are equivalent to (25)-(27),

$$
\left(\begin{array}{l}
M_{1}(0, t) \\
M_{2}(1, t) \\
M_{3}(0, t)
\end{array}\right)=\left(\begin{array}{ccc}
0 & K_{12} & 0 \\
K_{21} & 0 & K_{23} \\
0 & K_{32} & 0
\end{array}\right)\left(\begin{array}{l}
M_{1}(1, t) \\
M_{2}(0, t) \\
M_{3}(1, t)
\end{array}\right),
$$

where $K_{12}=f\left(K_{32}\right)=\frac{\rho^{*}\left(1-K_{32}\right)}{a^{*}}$.

The interest of the feedback laws $c_{0}(t)$ and $c_{1}(t)$ leads in the equivalent form (28) in Riemann coordinates, for which the stability analysis could be studied by applying our main result. Checking the assumptions of Theorem 1 allows to compute suitable tuning parameters $K_{21}, K_{23}$ and $K_{32}$. Moreover note that the controllers $c_{0}(t)$ and $c_{1}(t)$ do not depend on all the state $(\bar{u}, \bar{\rho}, \bar{p})^{\top}$, but depend on some boundary values, namely $\bar{p}(0, t), \bar{p}(1, t)$ and $\bar{\rho}(1, t)$. The proof of Proposition 1 is available in [17].

\section{Boundary condition synthesis based on singular perturba- tion method}

According to Section II, the reduced subsystem for gas transport system is computed as follows,

$$
\bar{M}_{1 t}+u^{*} \bar{M}_{1 x}=0,
$$

with the boundary condition

$$
\bar{M}_{1}(0, t)=K_{r} \bar{M}_{1}(1, t),
$$

where $K_{r}=\frac{\rho^{*}\left(1-K_{32}\right) K_{21}}{a^{*}\left(1-K_{23} K_{32}\right)}$.

Due to Proposition 1 in [18], the reduced subsystem (29) and (30) is convergent in finite time $T$ if the boundary condition $K_{r}=0$. Assuming $1-K_{23} K_{32} \neq 0$, since $K_{32} \neq 1$ in Proposition 1 , it holds $K_{r}=0$ as soon as $K_{21}=0$. The boundary condition matrix $K$ in (28) becomes $K=\left(\begin{array}{ccc}0 \frac{\rho^{*}\left(1-K_{32}\right)}{a^{*}} & 0 \\ 0 & 0 & K_{23} \\ 0 & K_{32} & 0\end{array}\right)$. 
To ensure $\rho_{1}(K)<1$, it is sufficient to choose $\|K\|<1$. In order to decrease the control cost, we can minimize $\|K\|$ that is equivalent to minimize $K_{32}^{2}+\left(\frac{\rho^{*}\left(1-K_{32}\right)}{a^{*}}\right)^{2}+K_{23}^{2}$. Let $K_{23}$ be zero. Computing the derivative of $K_{32}^{2}+\left(\frac{\rho^{*}\left(1-K_{32}\right)}{a^{*}}\right)^{2}$ with respect to $K_{32}$, we obtain $K_{32}=\frac{\rho^{* 2}}{\rho^{* 2}+a^{* 2}}$. Therefore the control actions become $c_{0}(t)=c_{0}^{*}-\frac{\frac{s\left(a^{* 2}+2 \rho^{* 2}\right)}{\sigma a^{* 3} \rho^{*}}-c_{0}^{*}}{p^{*}-p_{i n}} \bar{p}(0, t)$ and $c_{1}(t)=c_{1}^{*}+\frac{\frac{s}{\sigma a^{*} \rho^{*}}+c_{1}^{*}}{p_{\text {out }}-p^{*}} \bar{p}(1, t)$.

c) Numerical results: Let us consider the following values for numerical simulation: $a^{*}=(200,150,100), u^{*}=10, \rho^{*}=2$, $K=10^{-5}\left(\begin{array}{cccc}0 & 600 & 0 \\ 0 & 0 & 0 \\ 0 & 4 & 0\end{array}\right)$. The time evolution of the solution $\bar{M}_{1}$ for the reduced subsystem (29) with $K_{r}=0$ is shown in Figure 1a. It is observed that $\bar{M}_{1}$ converges to the origin in finite time. Time evolution of $\eta$ in Figure $1 \mathrm{~b}$ shows that the error between the full system and the reduced subsystem is close to 0 as time increases. Table II gives the evolutions of $\|\eta(., t=0.1)\|_{L^{2}}^{2}$ and of $\int_{0}^{1}\|\delta(., t)\|_{L^{2}}^{2} d t$. It is found that the values are near zero and increase when $\epsilon$ increases, as expected from Theorem 1.

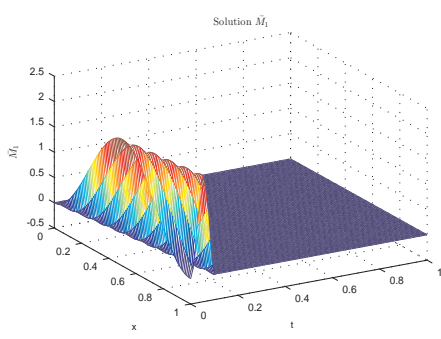

(a) Time evolution of $\bar{M}_{1}$

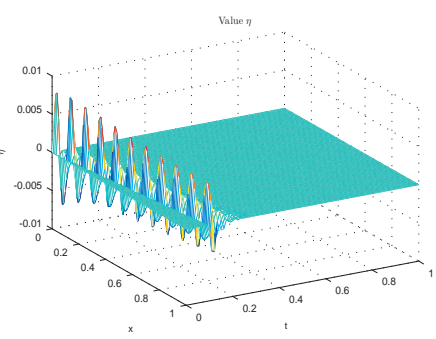

(b) Time evolution of $\eta$
Fig. 1: Time evolutions of $\bar{M}_{1}$ and $\eta$

\begin{tabular}{|c|c|c|c|}
\hline$\epsilon=\frac{u^{*}}{a^{*}}$ & $\frac{10}{200}$ & $\frac{10}{150}$ & $\frac{10}{100}$ \\
\hline$\|\eta(., t=0.1)\|_{L^{2}}^{2}$ & $4.0 \times 10^{-7}$ & $9.9 \times 10^{-7}$ & $2.6 \times 10^{-6}$ \\
\hline $\int_{0}^{1}\left\|\delta_{1}(., t)\right\|_{L^{2}}^{2} d t$ & $3.7 \times 10^{-19}$ & $3.1 \times 10^{-11}$ & $1.1 \times 10^{-4}$ \\
\hline $\int_{0}^{1}\left\|\delta_{2}(., t)\right\|_{L^{2}}^{2} d t$ & $1.1 \times 10^{-14}$ & $1.1 \times 10^{-11}$ & $3.4 \times 10^{-7}$ \\
\hline
\end{tabular}

TABLE II: Evolutions of square of $L^{2}$-norm of $\eta$ and of time integral of square of $L^{2}$-norm $\delta$ for different $\epsilon$

\section{CONCLUSION}

This paper is concerned with a class of singularly perturbed linear hyperbolic systems with source term which depends on the perturbation parameter. The hetero-directional transport velocities depend on $\epsilon$ as well as the boundary conditions. Under some assumptions and the condition $\rho_{1}(G(0))<1$, the approximation of the solution of the full system by that of the reduced subsystem has been established in Theorem 1. An academic example has been used to illustrate the main result. Furthermore, a new boundary control synthesis has been given with an application of gas flow transport model where the slow dynamics is convergent in finite time.

For the future work, it would be interesting to study a physical application with small source term which vanishes when the perturbation parameter tends to zero.

\section{REFERENCES}

[1] G. Bastin, J-M. Coron, and B. d'Andréa-Novel. Using hyperbolic systems of balance laws for modeling, control and stability analysis of physical networks. In Lecture notes for the Pre-Congress Workshop on Complex Embedded and Networked Control Systems, Seoul, Korea, 2008. 17th IFAC Word Congress.

[2] F. Castillo, E. Witrant, and L. Dugard. Contrôle de température dans un flux de Poiseuille. In IEEE Conférence Internationale Francophone d'Automatique, Grenoble, France, 2012.

[3] J-M. Coron, G. Bastin, and B. d'Andréa-Novel. Dissipative boundary conditions for one-dimensional nonlinear hyperbolic systems. SIAM Journal on Control and Optimization, 47(3):1460-1498, 2008.

[4] M. Dick, M. Gugat, and G. Leugering. Classical solutions and feedback stabilization for the gas flow in a sequence of pipes. Networks and Heterogeneous Media, 5(4):691-709, 2010.

[5] M. Gugat. Boundary feedback stabilization of the telegraph equation: Decay rates for vanishing damping term. Systems \& Control Letters, 66:72-84, 2014.

[6] M. Gugat and M. Herty. Existence of classical solutions and feedback stabilization for the flow in gas networks. ESAIM: Control, Optimisation and Calculus of Variations, 17:20-51, 2011.

[7] B. Haut and G. Bastin. A second order model of road junctions in fluid models of traffic networks. Networks and Heterogeneous Media, 2(2):227-253, 2007.

[8] H. K. Khalil. Nonlinear systems. Prentice-Hall, 1996.

[9] P.V. Kokotović and A.H. Haddad. Singular perturbations of a class of time optimal controls. IEEE Transactions on Automatic Control, 20:163-164, 1975.

[10] P.V. Kokotović, R.E. O'Malley, and P. Sannuti. Singular perturbation and order reduction in control theory-an overview. Automatica, 12:123-132, 1976.

[11] P.V. Kokotović and P. Sannuti. Singular perturbation method for reducing the model order in optimal control design. IEEE Transactions on Automatic Control, 13:377-384, 1968.

[12] P.V. Kokotović and R.A. Yackel. Singular perturbation of linear regulators: basic theorems. IEEE Transactions on Automatic Control, 17:29-37, 1972

[13] A. Saberi and H.K. Khalil. Quadratic-type Lyapunov functions for singularly perturbed systems. IEEE Transactions on Automatic Control, AC-29(6):542-550, 1984

[14] L.F. Shampine. Solving hyperbolic PDEs in Matlab. Applied Numerical Analysis and Computational Mathematics, 2:346-358, 2005.

[15] L.F. Shampine. Two-step Lax-Friedrichs method. Applied Mathematics Letters, 18:1134-1136, 2005.

[16] Y. Tang. Stability analysis and Tikhonov approximation for linear singularly perturbed hyperbolic systems. PhD thesis, Université Grenoble Alpes, 2015.

[17] Y. Tang, C. Prieur, and A. Girard. Singular perturbation approximation of linear hyperbolic systems of balance laws (full version). https://hal.archives-ouvertes.fr/hal-01175825.

[18] Y. Tang, C. Prieur, and A. Girard. Boundary control synthesis for hyperbolic systems: a singular perturbation approach. In Conference on Decision and Control, pages 2840-2845, Los Angeles, USA, 2014.

[19] Y. Tang, C. Prieur, and A. Girard. Tikhonov theorem for linear hyperbolic systems. Automatica, 57:1-10, 2015.

[20] D. E. Winterbone. Theory of Engine Manifold Design: Wave Action Methods for IC Engines. Society of Automotive Engineers. Inc., 2000.

[21] E. Witrant, K. Johansson, and the HynX team. Air flow modelling in deep wells: application to mining ventilation. In IEEE Conference on Automation Science and Engineering, pages 845-850, Arlington, VA, 2008. 